\title{
Space Station Attitude Disturbance Arising from Internal Motions
}

\author{
Patrick J. McNally and N. Harris McClamroch \\ Department of Aerospace Engineering \\ The University of Michigan \\ Ann Arbor, Michigan 48109
}

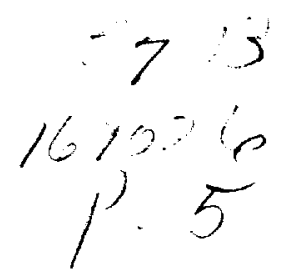

Abstract

A source of space station attitude disturbances is identified. The attitude disturbance is driven by internal space station motions and is a direct result of conservation of angular momentum. Three examples are used to illustrate the effect: a planar three link system, rigid carrier body with two moveable masses, and a nonplanar five link system. Simulation results are given to show the magnitude of the attitude change in each example. Factors which accentuate or attenuate this disturbance effect are discussed.

\section{Introduction}

A variety of nonclassical, inherently nonlinear dynamic modelling and control problems have been investigated recently. These investigations have revealed new possibilities for controlling certain systems and new explanations for certain sources of disturbances. These ideas are directly related to control and distubance analyses for the space station.

For example, a planar multilink system can be reoriented to an arbitrary attitude using only internal motions [1], [2]. Internal motions are executed in the shape space, defined by the relative angles of the links, to achieve a desired change in the absolute orientation. This effect can be extended to nonplanar multibody systems (as shown in an example given later) to allow arbitrary reorientation.

An example [3] of a rigid body with point mass oscillators also illustrates this effect. In this example, point masses move in slots illustrates this effect. The mondel was motivated by the attitude drift
This paper illustrates these effects for the space station through several examples. These examples serve to illustrate the magnitude of this disturbance effect, as well as to distinguish this disturance from other disturbances such as atmospheric drag and solar wind. The emphasis in this paper is on internal motions of the space station, driven externally or internally, which can result in an attitude change of the space station.

\section{Space Station and Large Space Structures}

Design of the space station (or other large spacecraft structures) is presented with competing requirements. Of particular focus here is the requirement to maintain stable pointing of the overall structure in the presence of additional requirements to point antennas and payloads, stabilize appendages, and conduct internal operations. For instance, the momentum management and attitude control system for the space station must provide space station attitude control within 5 deg of the local vertical and local horizontal lines, with an attitude rate boundary of $0.02 \mathrm{deg} / \mathrm{sec}$. The design goal for nominal operation is to maintain the station attitude excursion to less than $0.2 \mathrm{deg}$ from the average equilibrium attitude and the total attitude within $5 \mathrm{deg}$ of the local vertical and local horizontal lines. The attitude excursion is relaxed to 1 deg during attitude seeking [6]. Nominal operations, however, include astronaut activities, solar panel actuation. antenna actuation, and many other potential disturbances.

We are interested in exploring a particular class of disturbances that can modify the attitude of the space station. Some elements of the space station that may produce such attitude disturbance effects include:

1) Motions of flexible bodies, such as solar arrays, connecting beam structures, and laboratory modules, excited by external or 
into place to define new overall configurations

3) Internal motions of astronauts, servicing robots, centrifuges, and circulating pumps. For the space station, a servicing robot has been discussed that would traverse the beam sections of the space station.

Effects of the above three classes of configuration changes are illustrated by three examples.

\section{Example 1: PLanar Three Link Model}

Consider a planar model of a space station with central body and two rigid appendages (figure 1). Appendages could represent mechanical links, such as the space station beta joints, or they could represent a lumped parameter approximation of a large flexible structure. The model is characterized by (1) the distance between each link center of mass and the connecting link hinge

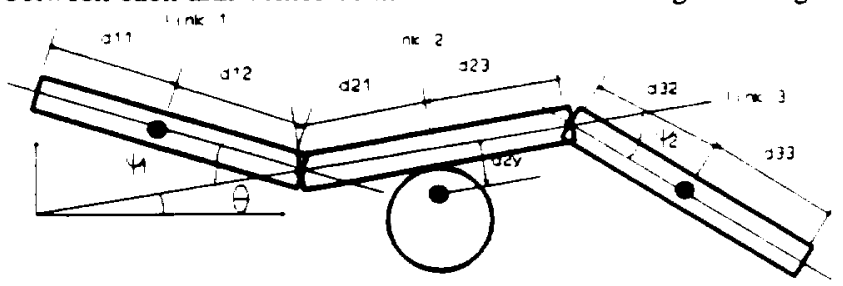

Figure 1: Planar Three Link System

points, (2) the mass and inertia of each link. The appendages are restricted to move as rotational links only. The configuration space is given by the two hinge angles $\left(\psi_{1}, \psi_{2}\right)$ and the overall orientation of one of the links, $\theta$. The shape space is given by the two hinge angles. This type of dynamic system has received much attention in the literature [1], [2], [7], [8]. We are interested here in a modification of the special kinematic case presented in [7]. Our modification includes an offset of the middle link center of mass from the line connecting the two hinge points. The model can also be extended to include additional links; however, three links are sufficient (and necessary) to illustrate the attitude change effect.

The primary relation of importance for our discussion is the angular momentum expression for the system. Since we are considering zero external torque on the system, angular momentum is constant throughout the motion of the appendages. The angular momentum $\mu$ is written as:

$$
\mu=J\left(\nabla_{1}, \nabla_{2}\right) \dot{\theta}+N_{3}\left(\downarrow_{1}, \nabla_{2}\right) \dot{\phi}_{1}+N_{2}\left(\nabla_{1}, \downarrow_{2}\right) \dot{\nabla}_{2}
$$

where

$$
\begin{aligned}
& J\left(\downarrow_{1}, \psi_{2}\right)=k_{1}+k_{2} \cos \left(\psi_{1}\right)+k_{3} \cos \left(\psi_{2}\right)+k_{6} \cos \left(\downarrow_{1}+\psi_{2}\right) \\
& +k_{5} \sin \left(\psi_{2}\right)+k_{8} \sin \left(\psi_{2}\right) \\
& N_{1}\left(\omega_{1}, \phi_{2}\right)=k_{1}+k_{1} \cos \left(\phi_{2}\right)+k_{1} \cos \left(\phi_{2}\right) \\
& +k_{10} \cos \left(\nabla_{1}+\phi_{2}\right)+k_{11} \sin \left(t_{2}\right) \\
& N_{2}\left(\nabla_{1}, \downarrow_{2}\right)=k_{12}+k_{1}, \cos \left(\downarrow_{1}\right)+k_{16} \cos \left(\nabla_{2}\right) \\
& +k_{15} \cos \left(\nabla_{1}+\nabla_{2}\right)+k_{16} \sin \left(\nabla_{2}\right)
\end{aligned}
$$

The constants $k_{1}$ through $k_{16}$ are functions of the link kinematic parameters only [8]. Note that the angular momentum is not a function of the orientation angle. The Lagrangian function constructed for this system would show that $\theta$ is ignorable. We assume that the appendages are excited according to:

Further, this excitation is persistent for a long period of time (several orbital periods). The excitation is characterized by (1)

$$
\begin{aligned}
& \phi_{1}(t)=a \sin \left(\frac{2 \pi t}{T}+\phi_{1}\right)+\psi_{10} \\
& \phi_{2}(t)=a \sin \left(\frac{2 \pi t}{T}+\phi_{2}\right)+\psi_{20}
\end{aligned}
$$

a phase difference between the two appendages $\left(\phi_{1} \neq \phi_{2}\right)$, and (2) a nonzero mean value $\left(\psi_{10} \neq 0\right.$ and $\left.\psi_{20} \neq 0\right)$. The importance of these two assumptions is explained later.

\begin{tabular}{|c|c|c|}
\hline$\because x$ & $-1 * 2$ & ares? \\
\hline $\begin{array}{l}a 11=20 \mathrm{~m} \\
0: 2=20 \pi \\
m=3000 \mathrm{~kg} \\
1=230.5 \mathrm{kg-m}=2\end{array}$ & $\begin{array}{l}\mathrm{a} 21=20 \mathrm{~m} \\
023=20 \mathrm{~m} \\
02 \mathrm{dr}=10 \mathrm{~m} \\
\mathrm{mz}=5000 \mathrm{k0} \\
12=200.6 \mathrm{~kg}-\mathrm{mr} 2\end{array}$ & $\begin{array}{l}a 32=20 \mathrm{~m} \\
033=20 \mathrm{~m} \\
033=3000 \times 8 \\
m 3=23 \times .5 \times-m 2\end{array}$ \\
\hline
\end{tabular}

In order to make our results concrete, a set of parameters is selected for this example, representing an approximation of a large space structure with two flexible appendages (see table 1). For this example, $\left(\phi_{1}, \phi_{2}\right)=(0.0, \pi / 2),\left(\psi_{10}, \psi_{30}\right)=(\pi / 8, \pi / 8)$, and $\alpha=\pi / 8$ rad. For this system, simulation results clearly indicate that there is a small but steady drift in the orientation angle of the base link (figure 2)

Table 1: Parameters for 3 Link System

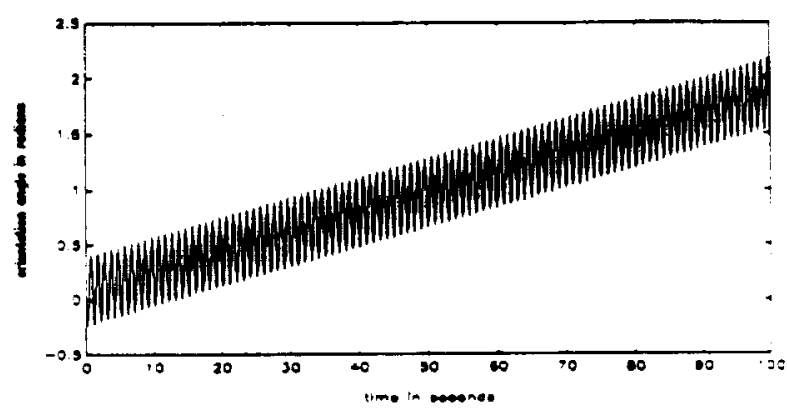

Figure 2: Orientation Change for 3 Link System

\section{Example 2: Rigid Body with Moveable Point Masses}

Consider a model of a rigid space station module with two internal moveable masses, for instance representing astronaut motions, mobile robot motions, or a centrifuge facility (figure 3 ). This model is an adaptation of a model originally presented in [3]. The model is characterized by (1) the path along which the masses move, and (2) the carrier body inertia matrix and the masses of each element. For this model, $R \in S O(3)$ represents the orientation of the carrier body with respect to the inerial frame and $q_{1}$ and $q_{2}$ are the position vectors of the oscillators with respect to the carrier-fixed frame. Also, 0 is the angular velocity of the body in the carrier frame, $b$ is the inertia matrix of the carrier body, and ( $\left.{ }^{\wedge}\right)$ represents the skew symmetric matrix formed by the components of () under the standard isomorphism ${ }^{\wedge}: R^{3} \rightarrow$ so(3) given by:

$$
\left(x_{1}, x_{2}, x_{3}\right) \mapsto\left|\begin{array}{ccc}
0 & -x_{1} & x_{2} \\
x_{3} & 0 & -x_{2} \\
-x_{2} & x_{1} & 0
\end{array}\right|
$$

The important relation here is the angular momentum expression. 
Table 2: Parameters for Cylinder with Moveable Masses

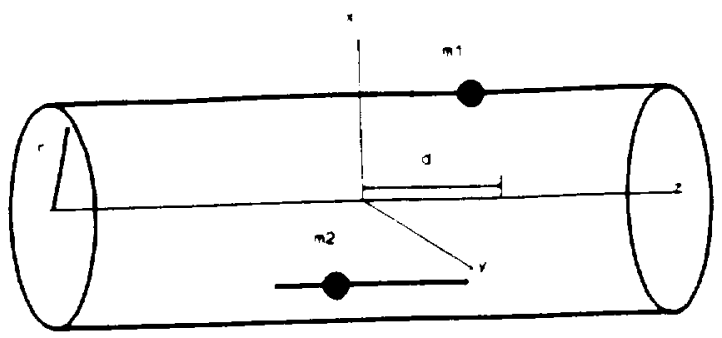

Figure 3: Rigid Central Body with 2 Moveable Masses

$$
\mu=R\left(I_{0}+\Delta I_{0}\right) Q+D_{1} \phi_{1}+D_{2} \Phi_{2}
$$

where

$$
\begin{gathered}
\Delta I_{0}=-m\left[e_{2} q_{1}^{2}+e_{2} q_{2}^{2}-\left(e_{2} q_{1}+e_{2} q_{2}\right)^{2}\right] \\
D_{1}=m\left[\left(1-c_{2}\right) e_{2} q_{1}-c_{1} e_{2} q_{2}\right] \\
D_{2}=m\left[-e_{1} e_{2} q_{2}+e_{2}\left(1-e_{2}\right) q_{2}\right] \\
c_{1}=\frac{m_{1}}{m} \\
m=m_{0}+m_{1}+m_{2}
\end{gathered}
$$

\begin{tabular}{|c|c|c|}
\hline Exingor & Mass" & nose. 2 \\
\hline 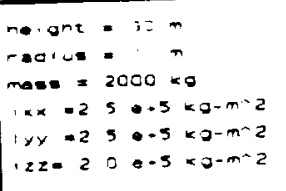 & $\begin{array}{l}m=90 \times 0 \\
91=50 \\
9 n \cdot 1=00 \\
a=10 m\end{array}$ & $\begin{array}{l}-2=100=0 \\
-2=5=0 \\
\text { pn12=157 }\end{array}$ \\
\hline
\end{tabular}

Consider again zero initial angular momentum. The body angular velocity vector is given by:

$$
Q=-I_{\text {lock }}^{-1}\left(D_{1} \phi_{2}+D_{2} \phi_{2}\right)
$$

where

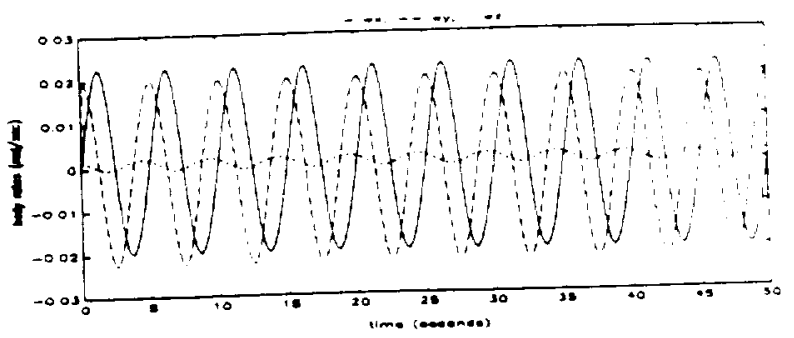

Figure 4: Body Rates for Rigid Body with Moveable Masses

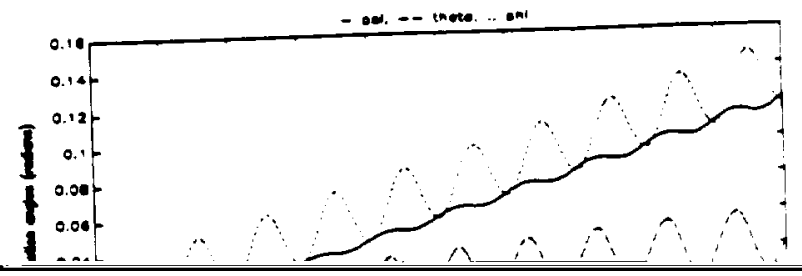




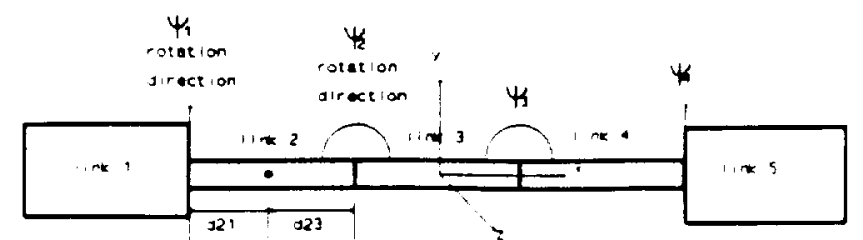

Figure 6: Five Link Nonplanar System

A sequence of three major motions is performed. Each motion segment consists of a movement of the two coplanar hinges while the other two hinges are held fixed. For simplicity, we choose motions that consist of square paths in the shape space. The entire sequence consists of a segment using the inner joints, then the outer joints, and finally the inner joints again (figure 7). Parameters for this example are shown in table 3.

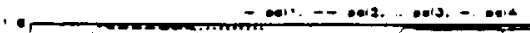

are integrated to obtain the base body attitude, expressed in Euler coordinates:

$$
\begin{gathered}
\dot{\phi}=-\omega_{y} \frac{\sin (\phi)}{\sin (\theta)} \\
\dot{\theta}=\omega_{y} \cos (\phi) \\
\dot{\phi}=-\omega_{y} \frac{\sin (\phi)}{\tan (\theta)}+\omega_{y}
\end{gathered}
$$

Several cycles of the joints were used in order to illustrate the orientation change. The resulting motion of the orientation angles is shown in figure 8 .

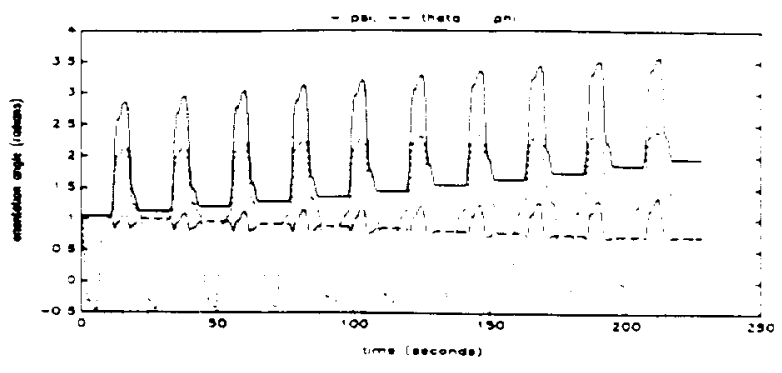

Figure 8: Attitude Drift for Five Link Nonplanar System

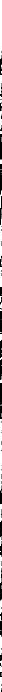

14 


$$
\Delta \theta=\iint\left(\frac{\partial f_{1}}{\partial \psi_{2}}-\frac{\partial f_{2}}{\partial \phi_{1}}\right) d \psi_{2} d \phi_{2}
$$

where

$$
\begin{aligned}
& E_{1}=\frac{N_{2}}{J} \\
& E_{2}=\frac{N_{2}}{J}
\end{aligned}
$$

The integrand of this function can be plotted versus the joint angles for the parameters used in example 1 (figure 9). For example 2, a similar result can be obtained where the body axis rate components are found as a function of the two mass incremental motions. From the function shown in figure 9 , it is apparent that motions which contain an area with nonzero integral will result in an orientation change.

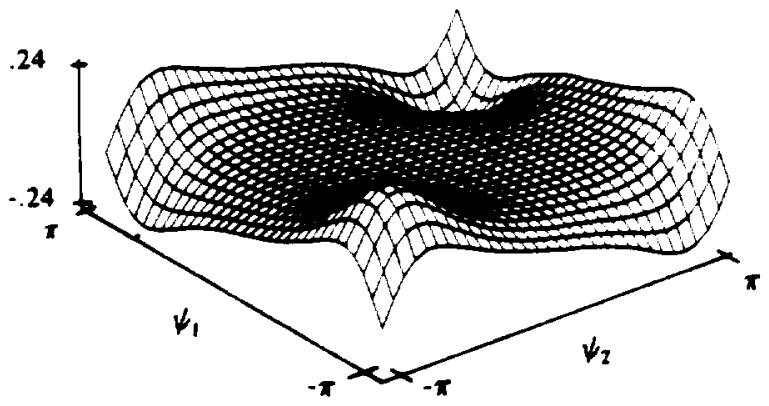

Figure 9: Integrand of Equation 9 versus Joint Angles

For general space manipulator systems, paths of minimum and maximum disturbance can be analyzed according to an enhanced
Some planning for "controllable" motions like robot and astronaut paths can mitigate some of the disturbance effects. These might be analyzed using equation 9 or the enhanced disturbance map given in [11]. The investigation of attitude changes from internal motions is important to minimize fuel required to operate the momentum management system on the space station.

\section{References}

[1] M. Reyhanoglu, N.H. McClamroch, "Reorientation of Space Multibody Systems Maintaining Zero Angular Momentum," AJAA GNCC 1991, pp. 1330-1340

[2] M. Reyhanoglu and N. H. McClamroch, "Controllability and Stabilizability of Planar Multibody Systems with Angular Momentum Preserving Control Torques," 1991 American Control Conference, pp. $1102-1107$

[3] P.S. Krishnaprasad, R. Yang, W.P. Dayawansa, "Control Problems on Principal Bundles and Nonholonomic Mechanics." CDC 1991, pp. 1133-1138

[4] P.S. Krishnaprasad, "Geometric Phases and Optimal Reconfiguration for Multibody Systems," ACC 1990, pp. 24402444

[5] G. Walsh, S. Sastry, "On Reorienting Linked Rigid Bodies Using Intemal Motions," 1991 CDC, pp. 1190-1195

[6] H. H. Woo, H.D. Morgan, E. T. Falangas, "Momentum Management and Attitude Control Design for a Space Station," J. Guidance, Vol. 11, No. 1, Jan. - Feb. 1988, pp. 19-25

I7 N Sreenath. Y.G. Oh, P.S. Krishaprasad, J.E. Marsden, 


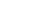

